\title{
Relay-Assisted Interference Network: Degrees of Freedom
}

\author{
Ramy Abdallah Tannious and Aria Nosratinia, Fellow, IEEE
}

\begin{abstract}
This paper investigates the degrees of freedom of the interference channel in the presence of a dedicated multiple-input multiple-output (MIMO) relay. The relay is used to manage the interference at the receivers. It is assumed that all nodes including the relay have channel state information only for their own links and that the relay has $M \geq K$ antennas in a $K$-user network. We pose the question: what is the benefit of exploiting the direct links from the source to destinations compared to a simpler two-hop strategy. To answer this question, we first establish the degrees of freedom of the interference channel with an MIMO relay, showing that a $K$-pair network with an MIMO relay has $\frac{K}{2}$ degrees of freedom. Thus, appropriate signaling in a two-hop scenario captures the degrees of freedom without the need for the direct links. We then consider more sophisticated encoding strategies in search of other ways to exploit the direct links. Using a number of hybrid encoding strategies, we obtain nonasymptotic achievable sum rates. We investigate the case where the relay (unlike other nodes) has access to abundant power, showing that when sources have power $P$ and the relay is allowed power proportional to $\mathcal{O}\left(P^{2}\right)$, the full degrees of freedom $K$ are available to the network.
\end{abstract}

Index Terms-Degrees of freedom (DOF), interference channel (IFC), relay channel, wireless networks.

\section{INTRODUCTION}

I $\mathrm{N}$ addition to historical significance in network information theory, a better understanding of the interference channel (IFC) [1] is becoming increasingly practically important, since many current wireless communication systems are interferencelimited. Examples include ad-hoc networks with peer-to-peer communications that lack infrastructure and hence transmission coordination, interference between adjacent networks in wireless LAN systems, as well as cognitive networks, where primary and secondary users transmit in the same band.

The capacity of the IFC in the most general case remains unknown; thus, a number of partial approaches for investigating the IFC have been pursued. One of the tools for understanding

Manuscript received November 19, 2008; revised August 26, 2011; accepted December 06, 2011. Date of publication January 31, 2012; date of current version May 15, 2012. This work was supported in part by the National Science Foundation under Grant CNS-0435429. The material in this paper was presented in part at the 2008 IEEE International Symposium on Information Theory.

R. A. Tannious was with The University of Texas at Dallas, Richardson, TX 75083 USA. He is now with Aviat Networks, Santa Clara, CA 95054 USA (e-mail: ramy@alumnimail.utdallas.edu).

A. Nosratinia is with the Department of Electrical Engineering, The University of Texas at Dallas, Richardson, TX 75083 USA (e-mail: aria@ utdallas.edu).

Communicated by S. Ulukus, Associate Editor for Communication Networks.

Color versions of one or more of the figures in this paper are available online at http://ieeexplore.ieee.org.

Digital Object Identifier 10.1109/TIT.2012.2184644 the behavior of multiterminal networks is the degrees of freedom (DOF), also known as the multiplexing gain or the pre-log factor, which characterizes the scaling behavior of a network throughput at high signal-to-noise ratios (SNRs). We formally define the DOF as follows [2]:

$$
\mathrm{DOF}=\lim _{P \rightarrow \infty} \frac{C_{s}}{\log \left(\frac{P}{\sigma^{2}}\right)}
$$

where $P$ is the power constraint at each source node, $\sigma^{2}$ is the noise variance at a destination, and $C_{s}$ is the network sum-rate capacity. For example, the maximum DOF of a two-user (singleantenna) Gaussian IFC is equal to one [3].

This paper investigates the effect of having a dedicated multiple-input multiple-output (MIMO) relay shared by several source-destination pairs on the DOF of such network. The main issue is whether with simple single-user decoding at the destinations, exploiting direct links is of a benefit.

Recent advances in network information theory have led to the characterization of the DOF of several networks. It is well known that the MIMO multiple access channel (MAC) and MIMO broadcast channel (BC) have full DOF [4], [5]. Thus, the DOF in the MIMO MAC and BC channels do not increase with transmit and receive cooperation, respectively.

Recently, the phenomenon of interference alignment has led to new results that characterize the DOF in various interference networks. The idea of interference alignment is for the transmissions to coordinate in such a manner such that at the receivers, the interference signals overlap in certain dimensions and, therefore, other dimensions are left interference free. Via interference alignment, in a $K$-user time-varying interference network $\frac{K}{2}$ DOF are achieved almost surely [6].

The first attempt to study the effect of relaying on the DOF of the interference network was performed in [3] and [7]. A rather negative result was obtained, showing that cooperation over fading links between the sources, between the destinations, or both, cannot improve the DOF of an interference network. On the other hand, if perfect cooperation between sources (destinations) is assumed, the network can mimic an MIMO system with antennas colocated at the transmitting (receiving) side as mentioned previously. In [8], the links between sources or between destination are considered having phase fading and it is shown that cooperation can help in increasing the throughput of a two-user IFC close to rates achieved by a $2 \times 2$ MIMO system. Considering distributed dedicated relays, Morgenshtern and Bölcskei [9] showed that the interference network can decouple. This is based on devising an amplify-and-forward two-hop strategy that utilize full (but local) CSI at the relays and subject to having the number of relays $m$ greater 
that $K^{3}$, where $K$ is the number of source-destination pairs. A similar decoupling can be achieved by fewer relays, specifically $m \geq K^{2}$ with the cost of having global CSI at the relays [10]. The DMT performance of this scheme was further analyzed in [11]. Finally, a two-hop network with MIMO relaying decouples into an MIMO-MAC followed by an MIMO-BC each achieving full DOF [12]; therefore, the DOF $\frac{K}{2}$ is achievable with a two-hop transmission.

The addition of an MIMO relay to an IFC (including direct links) gives rise to a network model that we denote the interference MIMO relay channel (IMRC). In this paper, we first establish the DOF of the Gaussian IMRC with the source and destination nodes having one antenna each. Achievability is demonstrated with a two-hop scheme, without exploiting the direct links from the sources to the destinations. The upper bound on the DOF of freedom is obtained by specializing the recently developed upper bounds in [13]. We establish that the IMRC has $\frac{K}{2} \mathrm{DOF}$.

We then take the investigation one step further to consider DOF beyond $\frac{K}{2}$. We devise new combinations of coding strategies that are inspired by the coding schemes used in relay channels, as well as MIMO MAC and MIMO BCs. These coding strategies attempt to exploit the direct links but at the same time manage the interference at the receivers using the MIMO relay. It is assumed that all nodes, including the relay, have only their own channel state information (CSI). We further consider the effect of the availability of abundant power at the relay. This is motivated by real-world scenarios where a single relay tower, with easy access to power, is assisting many mobiles. We wish to understand whether the devised coding scheme and the additional power at the relay can improve the DOF of the channel. Also, we investigate the minimum amount of power needed to impart maximum DOF to the network. We find that if the relay has $M \geq K$ antennas and power proportional to $\mathcal{O}\left(P^{2}\right)$, it can impart the maximum $K$ DOF to a $K$-user network whose users have power $\mathcal{O}(P)$, regardless of the number of users [cf., of our definition of DOF in (1)].

The remainder of this paper is organized as follows. Section II explains the notations used in this paper and provides the system model. Section III establishes the DOF of the $K$-user IMRC. Section IV states the main result of this paper and presents the detailed coding strategies that exploits the direct links and abundant power at the relay. We corroborate our analytical findings by numerical results in Section V. Finally, Section VI concludes this paper.

\section{SYSTEM MODEL}

Throughout this paper, lower-case and upper-case boldface letters denote vectors and matrices, respectively. The determinant of matrix $\mathbf{X}$, it's transpose, and Hermitian are denoted $\operatorname{det}(\mathbf{X}), \mathbf{X}^{\dagger}$, and $\mathbf{X}^{*}$, respectively. The norm of a vector $\mathbf{x}$ is denoted by $\|\mathbf{x}\| \cdot \log (\cdot)$ stands for the base- 2 logarithm. All rates are expressed in bits/channel use.

The IMRC is depicted in Fig. 1 . Nodes 1 and 2 attempt to communicate independent messages $W_{1}$ and $W_{2}$ to their respective receivers, possibly with help from the relay (node $R$ ). The relay is assumed to be equipped with $M$ antennas, where $M \geq 2$ while all other nodes have one antenna each. All links

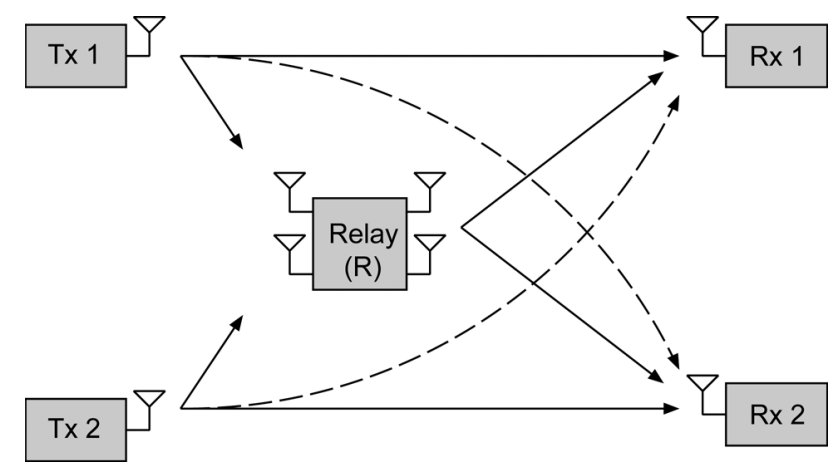

Fig. 1. Two-user IMRC.

are subject to flat fading which remains constant during the transmission period. The channels from the sources to their corresponding destinations, from the sources to the relay, and from the relay to destinations are denoted by the letters, $f, g$, and $h$, respectively. A subscript $a b$ is used to index the transmitting and receiving nodes, $a$ and $b$, respectively.

The input-output relation of a Gaussian IMRC is given by

$$
\begin{aligned}
y_{1} & =f_{11} x_{1}+\mathbf{h}_{R 1}^{\dagger} \mathbf{x}_{R}+f_{21} x_{2}+z_{1} \\
y_{2} & =f_{12} x_{1}+\mathbf{h}_{R 2}^{\dagger} \mathbf{x}_{R}+f_{22} x_{2}+z_{2} \\
y_{R} & =\mathbf{g}_{1 R} x_{1}+\mathbf{g}_{2 R} x_{2}+z_{R}
\end{aligned}
$$

where $y_{1}, y_{2}$, and $y_{R}$ are the channel outputs at receivers 1,2 and the relay, $x_{1}, x_{2}$, and $\mathbf{x}_{R}$ are the transmitted signals. The variables $z_{1}, z_{2}$, and $z_{R}$ denote zero-mean, unit-variance additive white Gaussian noises at the receivers. We assume individual block power constraints on the transmitting nodes. Nodes 1 and 2 have equal transmit power constraint of $P$, i.e.

$$
\sum_{i=1}^{n}\left\|x_{k}(i)\right\|^{2} \leq n P, \quad k=1,2
$$

where $i$ is the symbol index within a block of $n$ symbols.

We assume that the relay node has block power constraint $P_{R}$, which may be different from $P$ and will be specified in each instance in the sequel. The relay uses a decode-and-forward scheme [14] that includes linear pre-coding, in a manner to be explained shortly. The CSI knowledge assumptions are as follows. Transmitters 1 and 2 each have perfect knowledge about their own transmit-side CSI, while receivers 1 and 2 have perfect knowledge of their receive-side CSI. The relay is assumed to have knowledge of its incoming and outgoing links. The relay is assumed to operate in full-duplex mode, i.e., it can receive and transmit at the same time. Throughout this paper, we assume the input alphabets to be Gaussian. The average probability of error is defined as follows:

$$
P_{e}^{(n)}=\operatorname{Pr}\left[\left\{\hat{W}_{1} \neq W_{1}\right\} \cup\left\{\hat{W}_{2} \neq W_{2}\right\}\right]
$$

where $\hat{W}$ denotes an estimate of $W$. The rate of transmission from node $k$ is $R_{k}=\frac{\log Q_{k}}{n}$, where $Q_{k}$ is the size of the message transmitted by node $k$. A rate pair $\left(R_{1}, R_{2}\right)$ is said to be achievable for the IMRC if there exist a sequence of codes $\left(\left(2^{n R_{1}}, 2^{n R_{2}}\right), n\right)$ with average probability of error $P_{e}^{(n)} \rightarrow 0$ as $n \rightarrow \infty$. A $K$-user interference network with a single MIMO 
relay can be defined as a straightforward extension to the aforementioned model.

\section{DOF OF IMRC}

The first main result of this paper is as follows:

Theorem 1: The DOF of the interference MIMO relay network is $\frac{K}{2}$.

Proof: Achievability is established with a simple two-hop scheme. The first phase where the sources transmit the signals and the MIMO relay decodes is a MIMO MAC channel. It is well known that this channel achieves the full $K$ DOF. The second phase where the relay transmit to the receivers is a MIMO BC and again is know to achieve $K$ DOF. The transmission in two hops entails a penalty of one half in the DOF. Thus, $\frac{K}{2}$ DOF are achieved for the IMRC.

Now, the converse. A recent work [13] produced an elegant approach to find upper bounds on fully connected interference and $\mathrm{X}$ networks with relays and feedback. The upper bound on an $S \times R \times D$ fully connected network can be specialized to the network we study in this paper where $S, R$, and $D$ refer to the number of sources, relays, and distentions in the network, respectively. A fully connected network means that there is a message from every source to every destination. For completeness, we will first state the main result on the upper bound on the DOF of the $S \times R \times D$ network.

Theorem 2 [13]: If $\mathcal{D}$ represents the DOF region of the $S \times R \times D$ node $X$ network, then the total DOF can be upper bounded as follows:

$$
\max _{\left[\left(d_{i, j}\right)\right] \in \mathcal{D}} \sum_{j=1}^{S} \sum_{i=S+R+1}^{S+R+D} d_{i, j} \leq \frac{S D}{S+D-1} .
$$

Note that [13] derives upper bound not only on the DOF of the $S \times R \times D$ but on the whole DOF region. The interested reader is referred to [13] for further details.

Now for the $K$-user interference network, using the following corollary from [13] the exact DOF is obtained.

Corollary 1: Consider a fully connected $K$-user interference network with $R$ relays, where all the channel coefficients are time-varying/frequency-selective with values drawn randomly from a continuous distribution with support bounded below by a nonzero constant. Let all nodes be full-duplex allowing noisy transmitter/receiver cooperation. Also, let the source and relay nodes receive perfect feedback from all nodes. Then, the interference network has $\frac{K}{2}$ DOF.

The bounds in the previous theorem and corollary are applicable to the MIMO relay in the IMRC, because the proof of the converse assumes full cooperation between the distributed $R$ relay nodes (see observation 3 in [13]). Also, feedback and time/frequency selectivity of the channel do not reduce the DOF of the channel. Therefore, due to the matching achievability and converse results, the DOF of IMRC is established to be $\frac{K}{2}$.

\section{DOF BEYOND $K / 2$}

In the previous section, the DOF of the IFC with an MIMO relay was shown to be $\frac{K}{2}$, which is also achievable via a two-hop strategy. Therefore, the direct links do not contribute to the DOF of the channel. However, we will show that the DOF can be larger than $\frac{K}{2}$ by exploiting the direct links in addition to a more powerful MIMO relay.

We start by developing a coding strategy that uses the direct links and investigate, through the derived sum rate of the channel, the reason for the inefficiency of the direct links in improving the DOF. Then, the effect of abundant power at the relay on the DOF is studied.

\section{A. Coding Strategies and Achievable Rates}

The idea of the upcoming coding strategies is to use the relay in a way that minimizes the interference at the receivers. This task is highly nontrivial because the causality of the relay prohibits straight-forward interference cancellation. Therefore, sophisticated coding and power control strategies are needed to possibly manage the interference at the receivers.

Consider a transmission period of $B$ blocks, each of $n$ symbols. It is assumed that $n$ is sufficiently large to allow reliable decoding. Without loss of generality, at first a two-user network is considered. Nodes 1 and 2 send sequences of $B-1$ messages $\left(W_{1}(b)\right.$ and $\left.W_{2}(b)\right)$, respectively, over the channel in $n B$ transmissions, where $b$ denotes the block index, $b=1,2, \ldots, B-1$. The rate pair $\left(R_{1} \frac{B-1}{B}, R_{2} \frac{B-1}{B}\right)$ approaches $\left(R_{1}, R_{2}\right)$ as $B \rightarrow$ $\infty$.

\section{B. Encoding at the Sources}

The source uses the super-position block Markov encoding technique devised in [14]. In particular at any block $b$

$$
\begin{aligned}
& X_{1}^{(b)}=U_{1}+U_{1}^{\prime} \\
& X_{2}^{(b)}=U_{2}+U_{2}^{\prime}
\end{aligned}
$$

where $U_{1}$ and $U_{1}^{\prime}$ are i.i.d. Gaussian codebooks encoding the messages of the current and the previous blocks with powers $\chi(P)$ and $\psi(P)$, respectively, according to the power constraint

$$
\chi(P)+\psi(P)=P .
$$

Similar definitions hold for the signal components transmitted by node $2, U_{2}$ and $U_{2}^{\prime}$.

\section{Decoding and Re-Encoding at the Relay}

A space-division multiple-access (SDMA) approach is used to communicate between nodes 1, 2 and the MIMO relay. Therefore, both sources transmit simultaneously and the MIMO relay attempts decoding both signals. At the end block $b$, given that the relay decoded both messages $W_{1}(b-1)$ and $W_{2}(b-2)$ correctly, it can decode the messages $W_{1}(b)$ and $W_{2}(b)$ of both users while achieving a DOF $=2$. This can be achieved by a zero-forcing strategy, as long as the relay has no fewer antennas as the number of transmit nodes, and is made possible by the independence of the users' channels to the relay that is a result of spatial separation. The sum-rate constraint for correct decoding at the relay is given by [15, Sec 10.1]

$$
R_{1}+R_{2} \leq \log \operatorname{det}\left(\mathbf{I}_{2}+\mathbf{G K}_{x} \mathbf{G}^{*}\right)
$$

where $\mathbf{G}=\left[\mathbf{g}_{1 R} \mathbf{g}_{2 R}\right], \mathbf{K}_{x}=\operatorname{diag}(\chi(P), \chi(P))$, and $\mathbf{I}_{2}$ is the $2 \times 2$ identity matrix. 
Now, to the relay encoding strategies. Ideally, it would be desirable for the relay to cancel the entire interference at each receiver. However, due to causality, the relay can only cancel the interference arising from signals that it has already decoded. Thus, even if everything is accomplished perfectly, not all of the interferences will be canceled. The question is, if interferences cannot be fully removed, then how must the remaining interference be managed so that a good result may be obtained in terms of the DOF. This issue will be addressed in the sequel via power allocation policies at the sources and at the relay.

The channel from the relay to both destinations is similar to a Gaussian MIMO BC whose capacity region has been recently determined [16]. To help in canceling the interference, the relay uses a modified zero-forcing beamforming (ZF-BF) strategy [17]. ZF-BF achieves the maximum DOF of the sum-rate capacity of a Gaussian MIMO BC, although it is in general suboptimal compared to the capacity-achieving dirty-paper coding strategy. The relay constructs and transmits the following signal:

$$
\mathbf{x}_{R}^{(b)}=u_{1}^{\prime} \mathbf{t}_{\mathbf{1}}+u_{2}^{\prime} \mathbf{t}_{2}
$$

where $\mathbf{t}_{1}$ and $\mathbf{t}_{2}$ are $2 \times 1$ complex beamforming vectors. For simplicity, we assume the relay divides its power $P_{R}$ equally between the two signals components, i.e., $\left\|\mathbf{t}_{1}\right\|^{2}=\left\|\mathbf{t}_{2}\right\|^{2}=$ $\frac{P_{R}}{2 \psi(P)}$. Proper selection of beamforming vectors (magnitudes and phases) allows partial suppression of interference at the receivers as will be described later.

\section{Decoding at the Destinations}

Given the structure of the signal formed by the relay, we rewrite (2) and (3) as follows:

$$
\begin{aligned}
y_{1}^{(b)}=f_{11} u_{1} & +\left(f_{11}+\mathbf{h}_{R 1}^{\dagger} \mathbf{t}_{1}\right) u_{1}^{\prime} \\
& +\left(f_{21}+\mathbf{h}_{R 1}^{\dagger} \mathbf{t}_{2}\right) u_{2}^{\prime}+f_{21} u_{2}+z_{1} \\
y_{2}^{(b)}=f_{12} u_{1} & +\left(f_{12}+\mathbf{h}_{R 2}^{\dagger} \mathbf{t}_{1}\right) u_{1}^{\prime} \\
& +\left(f_{22}+\mathbf{h}_{R 2}^{\dagger} \mathbf{t}_{2}\right) u_{2}^{\prime}+f_{22} u_{2}+z_{2} .
\end{aligned}
$$

Therefore, the beamforming vectors at the relay $\mathbf{t}_{1}$ and $\mathbf{t}_{2}$ are selected such that $\mathbf{h}_{R 2}^{\dagger} \mathbf{t}_{1}=-f_{12}$ and $\mathbf{h}_{R 1}^{\dagger} \mathbf{t}_{2}=-f_{21}$. The derivation of $\mathbf{t}_{1}$ and $\mathbf{t}_{2}$ is discussed in the Appendix. This will cancel part of the interference seen by each receiver; thus, the received signals are modified to

$$
\begin{aligned}
& y_{1}^{(b)}=f_{11} u_{1}+\left(f_{11}+\mathbf{h}_{R 1}^{\dagger} \mathbf{t}_{1}\right) u_{1}^{\prime}+f_{21} u_{2}+z_{1} \\
& y_{2}^{(b)}=f_{12} u_{1}+\left(f_{22}+\mathbf{h}_{R 2}^{\dagger} \mathbf{t}_{2}\right) u_{2}^{\prime}+f_{22} u_{2}+z_{2} .
\end{aligned}
$$

Receivers 1 and 2 can use Willems's backward decoding to decode their intended signals [18]. Backward decoding imposes decoding delays; however, it simplifies the analysis compared to list decoding or window decoding [19]. Backward decoding starts from block $B$. The receivers have interference-free channels to decode $u_{1}^{(B-1)}$ and $u_{2}^{(B-1)}$. In block $B-1$, they presubtract the components of $u_{1}^{(B-1)}$ and $u_{2}^{(B-1)}$ before attempting to decode $u_{1}^{(B-2)}$ and $u_{2}^{(B-2)}$. Therefore, at any block $b$, the received signals can be further reduced to

$$
\begin{aligned}
& y_{1}^{(b)}=\left(f_{11}+\mathbf{h}_{R 1}^{\dagger} \mathbf{t}_{1}\right) u_{1}^{\prime}+f_{21} u_{2}+z_{1} \\
& y_{2}^{(b)}=f_{12} u_{1}+\left(f_{22}+\mathbf{h}_{R 2}^{\dagger} \mathbf{t}_{\mathbf{2}}\right) u_{2}^{\prime}+z_{2} .
\end{aligned}
$$

It is clear that channel does not have the typical form of an IFC

$$
y_{i}=h_{i i} x_{i}+h_{j i} x_{j}+z_{i}
$$

where $i, j \in\{1,2\}$ and $i \neq j$. Hence, we cannot further reduce the channel to a known form. Each receiver will attempt single user decoding, i.e., treating interference as noise, and thus can decode their respective messages $W_{1}$ and $W_{2}$ reliably if

$$
\begin{gathered}
R_{1} \leq \log \left(1+\frac{\left\|f_{11}\right\|^{2} \psi(P)+\left\|\mathbf{h}_{R 1}^{\dagger}\right\|^{2} \frac{P_{R}}{2}}{\left\|f_{21}\right\|^{2} \chi(P)+1}\right. \\
\left.+\frac{2 \alpha\left\|f_{11}\right\|\left\|\mathbf{h}_{R 1}^{\dagger}\right\| \sqrt{\psi(P) \frac{P_{R}}{2}}}{\left\|f_{21}\right\|^{2} \chi(P)+1}\right) \\
R_{2} \leq \log \left(1+\frac{\left\|f_{22}\right\|^{2} \chi(P)+\left\|\mathbf{h}_{R 2}^{\dagger}\right\|^{2} \frac{P_{R}}{2}}{\left\|f_{12}\right\|^{2} \psi(P)+1}\right. \\
\left.+\frac{2 \alpha\left\|f_{22}\right\|\left\|\mathbf{h}_{R 2}^{\dagger}\right\| \sqrt{\chi(P) \frac{P_{R}}{2}}}{\left\|f_{12}\right\|^{2} \psi(P)+1}\right)
\end{gathered}
$$

where $\alpha$ equals 0 when the CSI of the direct links, $f_{11}$ and $f_{22}$, is not available at the relay. The noncoherent addition of the signals coming from the sources and the relay entails a penalty in the achievable rate but does not affect the DOF. We can set $\alpha=1$ for perfect in-phase addition of the signals coming from the sources and the relay. ${ }^{1}$

We proceed to specify power allocation strategies, ranging from very simple to more sophisticated, and explore the corresponding achievable DOF. Let $\chi(P)=\psi(P)=\frac{P}{2}$ and $P_{R}=P$. According to this power allocation, the multiaccess part of the channel according to (10) achieves DOF $=2$. However, according to (19) and (20), the signal and interference have the same power order, and hence, a DOF $=0$ is achieved. Therefore, the DOF of the network in this case is zero. Clearly, this is not a desirable solution.

Now, consider an asymmetric power allocation policy characterized by $\chi(P)=\sqrt{P}, \psi(P)=|P-\sqrt{P}|$ and $P_{R}=P$. In other words, the cooperative information-also known as the resolution information-has a higher power than the information of the current block of transmission. It is clear that $\mathrm{DOF}=1$ is achieved on the multiaccess side of the channel. On the other hand, each of (19) and (20) provides a prelog factor of $\frac{1}{2}$ leading to a sum-rate $\mathrm{DOF}=1$ for the direct link with relaying. Therefore, an overall DOF $=1$ is achieved.

The impact of the aforementioned results are clearer when considered in the context of $K$ users. The previous coding strategies can easily be extended to an interference network where $K$ users transmit simultaneously and an MIMO relay having $M \geq K$ antennas helps all $K$ nodes in their transmission (see Fig. 2).

Having an MIMO relay in a network with $K$ source-destination pairs can have a large impact on the DOF either through a two-hop strategy or through the coding strategy developed in this section. Specifically, $\frac{K}{2}$ DOF are easily achieved compared to DOF of 1 with simple time-sharing strategy in the absence of the relay.

\footnotetext{
${ }^{1}$ The interested reader can refer to [20] for details on the capacity analysis of the full-duplex (a)synchronous relay channel with fixed and variable channel gains.
} 


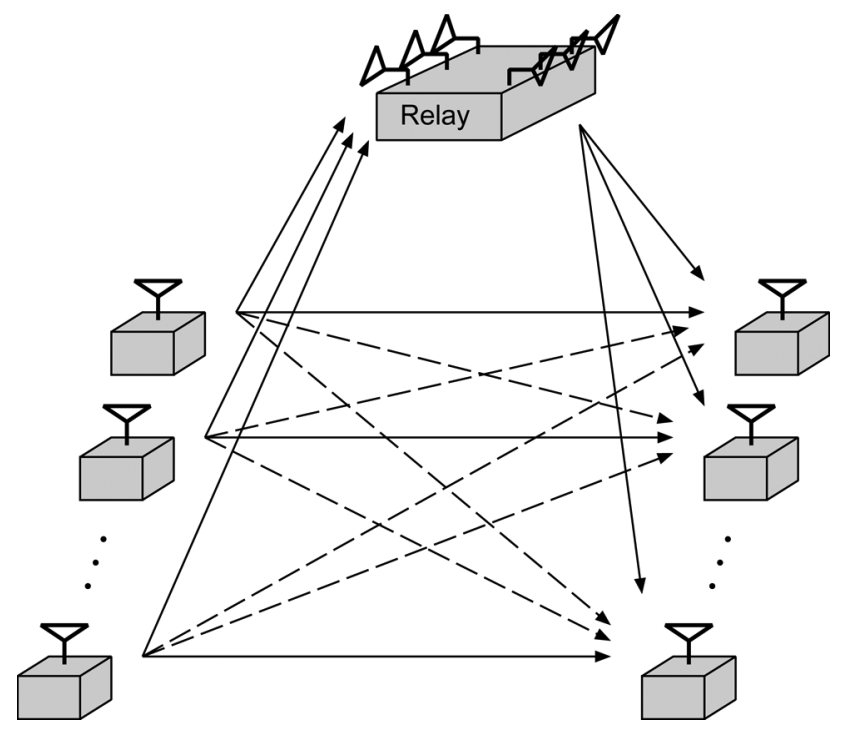

Fig. 2. Relay for $K$-user interference network.

So far, by exploiting the direct links, the DOF are no better than the simple two-hop strategy. Next, we explore a way to actually capture the whole $K$ DOF of the channel.

\section{E. Abundant Power at the Relay}

Assume $\chi(P)=\psi(P)=\frac{P}{2}$ at all source nodes while at the relay we have $P_{R}=P^{2}$ (or in general $\mathcal{O}\left(P^{2}\right)$ ). In this case, the network will achieve DOF of $K$, thanks to the precoding strategy employed by the relay, which allows the relay to avoid causing interference at any node.

Theorem 3: The $K$-user IMRC achieves $K$ (full) DOF (per the definition given in (1)), with the source nodes having each a per block power constraint of $P$ and the MIMO relay having a power constraint of $\mathcal{O}\left(P^{2}\right)$ and $M \geq K$ antennas.

Note that our definition of the DOF in (1) concentrates on the power of information-bearing nodes, thus allowing us to study the effect of abundant power at the relay for this special case.

Although the $K$ DOF are achieved with a relay that enjoys power proportional to $P^{2}$, it is noteworthy that the required relay power is independent on $K$.

\section{NUMERICAL RESULTS}

We corroborate the analysis by the following numerical example of an IMRC. The following setup is considered.

1) Two-user channel and the relay has two antennas, i.e., $K=$ $M=2$.

2) The noise variance at all nodes $\sigma^{2}=1$.

3) The magnitude of channel coefficients are selected as $f_{11}=2, f_{12}=0.75, f_{21}=0.75, f_{22}=2, \mathrm{~g}_{1 R}^{\dagger}=\left[\begin{array}{ll}2 & 0.8\end{array}\right]$, $\mathbf{h}_{2 R}^{\dagger}=\left[\begin{array}{ll}1.2 & 2\end{array}\right], \mathbf{h}_{R 1}^{\dagger}=\left[\begin{array}{ll}2 & 1\end{array}\right]$, and $\mathbf{h}_{R 2}^{\dagger}=\left[\begin{array}{ll}1 & 0.8\end{array}\right]$.

An IFC, without the MIMO relay, can be transformed into a well-known form in the literature known as the standard form (see e.g., [21]). The original IFC has the following standard form channel gains, under the assumption of unity noise variance at all nodes

$$
\begin{aligned}
& a_{12}=\left(\frac{f_{12}}{f_{11}}\right)^{2} \\
& a_{21}=\left(\frac{f_{21}}{f_{22}}\right)^{2} .
\end{aligned}
$$

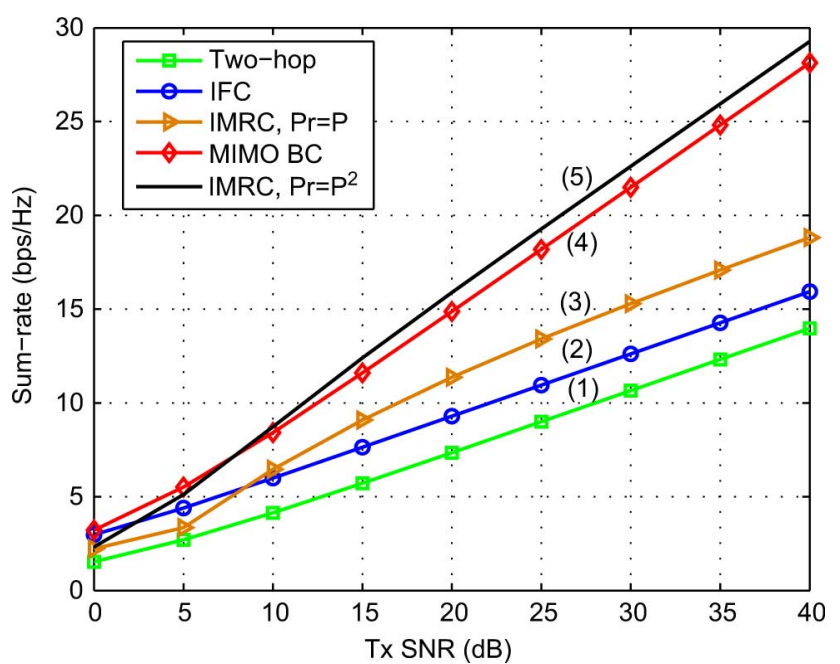

Fig. 3. Network throughput versus transmit SNR $P / \sigma^{2}$ of each source: twouser example.

The aforementioned values of the channel coefficients results in $a_{12}=a_{21}=0.14$. Thus, without the MIMO relay, the interference is considered weak/moderate. This is the case where the capacity region of the IFC is unknown and where a form of relaying will be of greater impact on the capacity [7].

Fig. 3 depicts the sum rate of five schemes. Curve (1) is the sum rate with a simple two-hop scheme. Curve (2) depicts the best known achievable sum rate for the IFC, with no relay present, using the Han-Kobayashi coding scheme. This scheme involves rate splitting, joint decoding at the receivers, and, moreover, includes a time-sharing random variable that switches between time-division transmission and simultaneous transmission by the source nodes. The cardinality of the time-sharing parameter is set to two, and furthermore, the power allocation of the rate-splitting scheme is optimized. This corresponds to curve 4 in [22]. Curve (3) is the computable sum rate of the IMRC with the coding strategy discussed in Section IV and under the asymmetric power allocation policy characterized by $\chi(P)=\sqrt{P}, \psi(P)=|P-\sqrt{P}|$ and $P_{R}=P$. Curve $(4)$ is the case where no relay is present and the two sources have ideal cooperation leading to an MIMO BC model. The optimal power allocation and, hence, the sum-rate capacity are computed according to Algorithm 2 in [23]. Finally, Curve (5) is the sum rate of the IMRC, however, under the assumption of abundant power at the relay, specifically, $\chi(P)=\psi(P)=\frac{P}{2}$ at the source nodes while at the relay we have $P_{R}=P^{2}$.

We emphasize, here, that for IMRC, we use independent decoding at the nodes and we do not fully optimize the power allocation strategies at the sources and the relay. The focus of this paper is on the DOF, and thus, the throughput optimization and analysis at finite SNR are outside the scope of this paper.

The following conclusions can be drawn from the figure.

1) The three lower curves share the same slope as the corresponding schemes have a DOF $=1$. The upper two curves also share another slope verifying that the IMRC with $P_{R}=P^{2}$ achieves the full DOF of the channel.

2) It is interesting to see that the fully optimized IFC as explained earlier can achieve higher throughput than the simple two-hop scheme; the fully optimized IFC in this 
figure has a total power equal to the total power of all the nodes in the IMRC, including the relay. The simple two-hop scheme does not involve optimal power allocation at the source nor joint decoding at the destinations.

3) While the coding strategy devised in this paper to exploit the direct links of the IMRC of Curve (3) shares the same DOF of IFC, but as it can be seen in the figure, this strategy leads to noticeable gains in the sum rate for medium and high SNR values over the IFC.

4) The IMRC scheme with abundant power at the relay, Curve (5), presents a substitute strategy for the cooperative MIMO, Curve (4). Cooperative MIMO is a scheme of interest in the wireless industry to improve the throughput for the uplink of cellular networks.

\section{DISCUSSION}

In this paper, we characterize the high-SNR sum-rate behavior of an IFC with an MIMO relay. First, we establish that the DOF of the IMRC to be only $\frac{K}{2}$. Therefore, in this case, exploiting the direct links does not provide significant throughput enhancement at high SNR. Then, we consider a more sophisticated coding scheme that exploits the direct links and the possibility of abundant power at the relay. We show that if the transmit/receive nodes have power proportional to $P$, and the relay has power proportional to $P^{2}$, all $K$ DOF of the channel become available. This result is achieved under modest channel knowledge assumption at the network nodes and with the assumption of single user decoding at the destinations.

While we consider the case of full-duplex relay, one can devise similar signaling strategies for the half-duplex case. However, the block-Markov coding is not required. A brief description of a possible coding scheme is given as follows. The sources transmit all the time. However, they divide each block of their transmission into two halves. Each source node transmits the same message in the two halves using i.i.d. Gaussian code books. During the second half, the relay transmits and manages the interference as discussed previously in the full-duplex case. At the destinations, the received signals at the first and second halves form two Gaussian parallel channels, the first sees interference while the other is interference free. It can be easily shown that the maximum DOF of this scheme is $\frac{K}{2}$. As we know from Theorem 1 , a two-hop strategy suffices to achieve a $\frac{K}{2}$ DOF. However, exploiting the direct links provides an increase in the throughput compared to two-hop communications for all SNRs.

Several directions naturally arise for future work. Our analysis concentrates on the high SNR behavior of the network throughput, thus many parameters of the IMRC can be further optimized for nonasymptotic SNR values. More complex coding/decoding techniques can also be employed, for example, a modified Han-Kobayashi scheme (in the presence of the MIMO relay) that combines rate-splitting, time sharing (time division multiplexing), relaying, and joint decoding at the receivers.

Finally, it is worth mentioning that, since the time of submission of this paper for publication, there has been a surge of research activity in the area of interference networks. In particular, the time/frequency nonselective IFC has been studied lately in more depth and a better understanding of its performance limits has been developed. The interested reader can refer to [24] and [25] and the references therein.

\section{APPENDIX}

For simplicity, we consider the two-user case. Denote by $\mathbf{t}_{1}=$ $\left[\begin{array}{ll}t_{11} & t_{12}\end{array}\right]$ and $\mathbf{t}_{2}=\left[\begin{array}{ll}t_{21} & t_{22}\end{array}\right]$ the beamforming vectors at the relay.

The following conditions govern the selection of the beamforming vector $\mathbf{t}_{1}$
1) $\left\|\mathbf{t}_{1}\right\|^{2}=\frac{P_{R}}{2 \psi(P)}$
2) $\mathbf{h}_{R 2}^{t} \mathbf{t}_{1}=-f_{12}$
3) $\Phi_{1}=\angle f_{11}=\angle \mathbf{h}_{R 1}^{t} \mathbf{t}_{1}$

where $\Phi_{1}$ is the phase of the (direct) channel between source node 1 and the intended destination node. Similarly, one can write the conditions for selecting $\mathbf{t}_{2}$.

The first condition is related to power scaling at the relay. The second condition is the one responsible to reduce the interference seen by the destination nodes. The third condition is optional; it is responsible for coherent combination of the desired signal component at the intended destination. It requires though global channel knowledge at the relay.

A closed-form solution for the three simultaneous conditions is not feasible. Instead, we assume the lack of the third condition which does not affect the DOF achieved by the coding scheme for IMRC explained in this paper and simplifies the channel knowledge requirements.

Solving for $\mathbf{t}_{2}$ and $\mathbf{t}_{2}$, one gets for $m \neq n$, and $m, n \in\{1,2\}$

$$
t_{m 1}=\frac{ \pm h_{R n, 2} \sqrt{-f_{m 2} \pm\left\|\mathbf{h}_{R n}\right\|^{2} \frac{P_{R}}{2 \psi(P)}}-f_{m 2} \mathbf{h}_{R m, 1}}{\left\|\mathbf{h}_{R n}\right\|^{2}}
$$

and

$$
t_{m 2}=-\frac{f_{m 2}}{h_{R n, 2}}-\frac{h_{R n, 1}}{h_{R n, 2}} t_{m 1}
$$

\section{REFERENCES}

[1] A. B. Carleial, "Interference channels," IEEE Trans. Inf. Theory, vol. 24, no. 1, pp. 60-70, Jan. 1978.

[2] K. Azarian, H. El Gamal, and P. Schniter, "On the achievable diversity-multiplexing tradeoff in half-duplex cooperative channels," IEEE Trans. Inf. Theory, vol. 51, no. 12, pp. 4152-4172, Dec. 2005.

[3] A. Høst-Madsen, "Capacity bounds for cooperative diversity," IEEE Trans. Inf. Theory, vol. 52, no. 4, pp. 1522-1544, Apr. 2006.

[4] D. Tse, P. Viswanath, and L. Zheng, "Diversity-multiplexing tradeoff in multiple-access channels," IEEE Trans. Inf. Theory, vol. 50, no. 9, pp. 1859-1874, Sep. 2004.

[5] S. Vishwanath, N. Jindal, and A. Goldsmith, "Duality, achievable rates, and sum-rate capacity of MIMO broadcast channels," IEEE Trans. Inf. Theory, vol. 49, no. 10, pp. 2895-2909, Oct. 2003.

[6] V. R. Cadambe and S. A. Jafar, "Interference alignment and degrees of freedom of the K-user interference channel," IEEE Trans. Inf. Theory, vol. 54, no. 8, pp. 3425-3441, Aug. 2008.

[7] A. Høst-Madsen and A. Nosratinia, "The multiplexing gain of wireless networks," in Proc. IEEE Int. Symp. Inf. Theory, Adelaide, Australia, Sep. 2005, pp. 2310-2314.

[8] C. T. K. Ng, N. Jindal, A. J. Goldsmith, and U. Mitra, "Capacity gain from two-transmitter and two-receiver cooperation," IEEE Trans. Inf. Theory, vol. 53, no. 10, pp. 3822-3827, Oct. 2007.

[9] V. I. Morgenshtern and H. Bölcskei, "Crystallization in large wireless networks," IEEE Trans. Inf. Theory, vol. 53, no. 10, pp. 3319-3349, Oct. 2007. 
[10] A. F. Dana and B. Hassibi, "On the power efficiency of sensory and ad hoc wireless networks," IEEE Trans. Inf. Theory, vol. 52, no. 7, pp. 2890-2914, Jul. 2006.

[11] C. K. Rao, "Asymptotic analysis of wireless systems with Rayleigh fading," Ph.D. dissertation, Cal. Inst. Technol., Pasadena, 2007.

[12] S. A. Jafar and S. Shamai, "Degrees of freedom region for the MIMO X channel," IEEE Trans. Inf. Theory, vol. 54, no. 1, pp. 151-170, Jan. 2008.

[13] V. R. Cadambe and S. A. Jafar, "Degrees of freedom of wireless networks with relays, feedback, co-operation and full duplex operation," IEEE Trans. Inf. Theory, vol. 55, no. 5, pp. 2334-2344, May 2009.

[14] T. Cover and A. E. Gamal, "Capacity theorems for the relay channel," IEEE Trans. Inf. Theory, vol. 25, no. 5, pp. 572-584, 1979.

[15] D. Tse and P. Viswanath, Fundamentals of Wireless Communication. New York: Cambridge Univ. Press, 2005.

[16] H. Weingarten, Y. Steinberg, and S. Shamai, "The capacity region of the Gaussian multiple-input multiple-output broadcast channel," IEEE Trans. Inf. Theory, vol. 52, no. 9, pp. 3936-3964, Sep. 2006.

[17] T. Yoo and A. Goldsmith, "On the optimality of multiantenna broadcast scheduling using zero-forcing beamforming," IEEE J. Sel. Areas Commun., vol. 24, no. 3, pp. 528-541, Mar. 2006.

[18] F. M. J. Willems, "Information-theoretical results for the discrete memoryless multiple access channel," Ph.D. dissertation, Katholieke Univ. Leuven, Leuven, Belgium, 1982.

[19] G. Kramer, M. Gastpar, and P. Gupta, "Cooperative strategies and capacity theorems for relay networks," IEEE Trans. Inf. Theory, vol. 51, no. 9, pp. 3037-3063, Sep. 2005.

[20] A. Høst-Madsen and J. Zhang, "Capacity bounds and power allocation for wireless relay channels," IEEE Trans. Inf. Theory, vol. 51, no. 6, pp. 2020-2040, Jun. 2005.

[21] G. Kramer, "Review of rate regions for interference channels," in Proc. Int. Zurich Semin. Commun., Feb. 2006, pp. 162-165.

[22] I. Sason, "On achievable rate regions for the Gaussian interference channel," IEEE Trans. Inf. Theory, vol. 50, no. 6, pp. 1345-1356, Jun. 2004.

[23] N. Jindal, W. Rhee, S. Vishwanath, S. A. Jafar, and A. Goldsmith, "Sum power iterative water-filling for multi-antenna Gaussian broadcast channels," IEEE Trans. Inf. Theory, vol. 51, no. 4, pp. 1570-1580, Apr. 2005.

[24] R. Etkin and E. Ordentlich, "On the degrees-of-freedom of the K-user Gaussian interference channel," in Proc. IEEE Int. Symp. Inf. Theory, Seoul, Korea, Jun.-Jul. 2009, pp. 1919-1923.
[25] A. S. Motahari, S. O. Gharan, M.-A. Maddah-Ali, and A. K. Khandani, "Real interference alignment: Exploiting the potential of single antenna systems," IEEE Trans. Inf. Theory, 2009 [Online]. Available: http:// arxiv.org/abs/0908.2282, submitted for publication

Ramy Abdallah Tannious received the B.S. and M.S. degrees from Alexandria University, Egypt, in 1999 and 2003, respectively, and the Ph.D. degree from the University of Texas at Dallas, TX, in 2008, all in electrical engineering. His dissertation work concerned the performance limits of communications in several wireless relay network architectures.

From 2000 to 2001, he served in the Signal Corps of the Egyptian Armed Forces. From 2001 to 2008, he held appointments at Alexandria University, SIDPEC Petrochemicals, University of South Florida, The University of Texas at Dallas, and Qualcomm, Inc. From May 2009 to August 2010, he was with the University of California Davis as a Post-doctoral Research Scientist in the Department of Electrical and Computer Engineering. Since September 2010, he has been with Aviat Networks, Santa Clara, CA, as a Senior R\&D Engineer developing the next generation of microwave radio products for wireless backhaul. His research interests lie in the broad area of wireless communication theory, with applications to relay networks and cooperative communications, cognitive radio networks, and body area networks. He has published over 15 journal and conference papers in these areas.

Aria Nosratinia (S'87-M'97-SM'04-F'10) is Jonsson Distinguished Professor at the Department of Electrical Engineering, the University of Texas at Dallas. He received his Ph.D. in Electrical and Computer Engineering from the University of Illinois at Urbana-Champaign in 1996. He has held visiting appointments at Princeton University, Rice University, and UCLA. His interests lie in the broad area of information theory and signal processing, with applications in wireless communications. He was associate editor for the IEEE TRANSACTIONS ON INFORMATION THEORY 2008-2011, was the secretary of the Information Theory Society in 2010, and served as treasurer for ISIT 2010 in Austin. He is currently an associated editor for the IEEE TRANSACTIONS ON WIRELESS COMMUNICATIONS. He has previously served as associate editor for the IEEE TRAnsactions on IMAge Processing, IEEE Signal Processing Letters, and IEEE Wireless Communications (Magazine). He has been the recipient of the National Science Foundation career award, and is a fellow of IEEE. 